\title{
Spatial and Temporal Patterns of Online Food Preferences
}

\author{
Claudia Wagner \\ GESIS \& University of Koblenz \\ claudia.wagner@gesis.org
}

\author{
Philipp Singer \\ Graz University of Technology \\ philipp.singer@tugraz.at
}

\author{
Markus Strohmaier \\ University of Koblenz \& GESIS \\ strohmaier@uni-koblenz.de
}

\begin{abstract}
Since food is one of the central elements of all human beings, a high interest exists in exploring temporal and spatial food and dietary patterns of humans. Predominantly, data for such investigations stem from consumer panels which continuously capture food consumption patterns from individuals and households. In this work we leverage data from a large online recipe platform which is frequently used in the German speaking regions in Europe and explore (i) the association between geographic proximity and shared food preferences and (ii) to what extent temporal information helps to predict the food preferences of users. Our results reveal that online food preferences of geographically closer regions are more similar than those of distant ones and show that specific types of ingredients are more popular on specific days of the week. The observed patterns can successfully be mapped to known real-world patterns which suggests that existing methods for the investigation of dietary and food patterns (e.g., consumer panels) may benefit from incorporating the vast amount of data generated by users browsing recipes on the Web.
\end{abstract}

\section{Categories and Subject Descriptors}

H.3.5 [Information Storage and Retrieval]: Online Information Services

\section{Keywords}

food, food preferences, recipes, ingredients, diet

\section{INTRODUCTION}

Food is one of the central elements of all human beings and it affects all aspects of human life including culture or health. Therefore a high interest exists in exploring temporal and spatial food and dietary patterns of humans. The data which enables such investigations usually stems from consumer panels which continuously produce longitudinal behavioral data on the consumption behavior of individuals

Copyright is held by the International World Wide Web Conference Committee (IW3C2). IW3C2 reserves the right to provide a hyperlink to the author's site if the Material is used in electronic media. WWW' 14 Companion, April 7-11, 2014, Seoul, Korea. ACM 978-1-4503-2745-9/14/04.

http://dx.doi.org/10.1145/2567948.2576951 . and households. However, generating this data is a timeconsuming and costly process and despite its strength it also suffers from limitations such as high drop-out rates, high latency or Howthorne effects.

In this work we explore the potential of unobtrusive research methods - i.e., methods using indirect ways to acquire data contrasting e.g., interviews - for exploring the dynamics of food preferences of individuals aggregated on a regional level. We use the number of requests for recipes on a recipe web site as a proxy for the food preferences of users and explore spatio-temporal patterns. Due to privacy concerns, we analyze the food preferences of groups of people living in the same geographic region rather than individual preferences. In this work we tackle both spatial and temporal aspects of patterns in online food preferences and address the following research questions: (i) Are online food preferences of geographically close regions more similar than those of distant regions? and (ii) To what extent do online food preferences change over time - i.e., during the course of a week?

\section{DATASET}

We analyze server log data from the largest online recipe platform in Austria, ichkoche.at. These logs reveal how frequently a recipe has been viewed in a certain region. The 184,296 recipes have been accessed by 1,695 different regions around 24 million times between August 2012 until November 2013. Even though the platform is from Austria, other German-speaking countries such as regions in Germany and Switzerland are prominently included (Bavaria, Zurich, Stuttgart, Hessen, Berlin, Worms, Bern and Saxony belong to the top 20 most active regions). Hence, we focus on data from the main German speaking regions in Europe - i.e., federal states in Austria, Germany and Switzerland.

Our data reveals that users tend to use the platform mostly on Mondays. During the week, usage decreases until Thursday and then mostly increases during the weekend. This finding is not in line with the findings of West et al. [3] who found that in the US people mainly search for recipes during the weekend. This indicates that differences may exist in the cooking and/or online recipe usage behavior of Europeans (or the German speaking population in Europa) and US citizens.

\section{SPATIAL PATTERNS}

One potential cause of shared online food preferences is geographical proximity since frequent communication and migration may explain the adoption of food preferences [1]. 


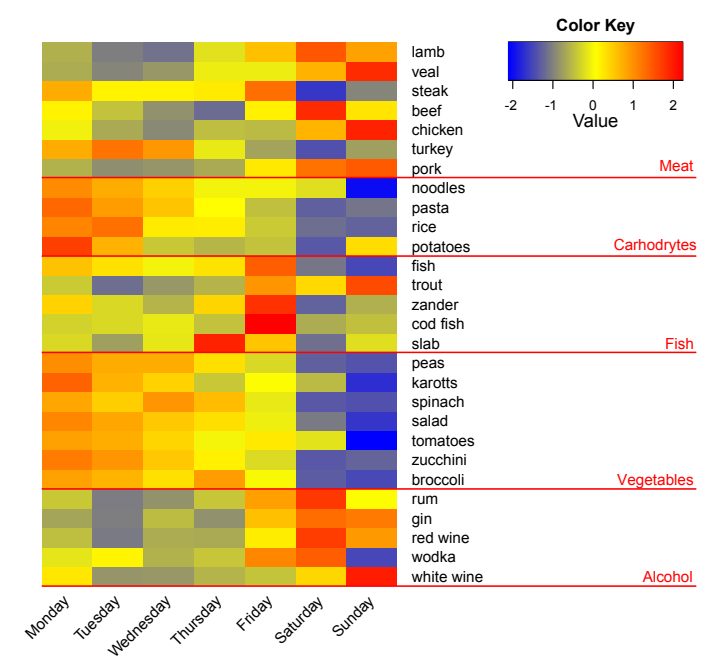

Figure 1: How popular are different ingredients over the course of a week? The plot shows z-scores of the request volume per weekday and the change rates of the request volume.

In the following, we want to test the hypothesis that geographically nearby regions are more similar regarding their food preferences than geographically distant locations.

We use the recipes/ingredients that have been requested from a certain region to estimate the shared online food preferences between regions. That means if two regions request the same recipes, they have a high recipe similarity. If two regions request recipes with similar ingredients, they have a high ingredients similarity though they might not access exactly the same recipes. We compute the recipe and ingredient similarity for all possible pairs of regions $(A, B)$ in the German speaking area of Europe using the Jaccard similarity $J_{t}=\frac{A_{t} \cap B_{t}}{A_{t} \cup B_{t}}$.

We test the hypothesis that geographical distant regions have more distinct online food preferences than geographic close regions by measuring the correlation between the geographical distance between regions and their recipe-similarity and ingredient similarity. We find a statistically significant negative correlation between the distance of regions and the recipe and ingredient similarities (Spearman's $\rho=-0.36$ for recipes and $\rho=-0.39$ for ingredients with $p<0.05$ ). This indicates that indeed regions which are geographically closer exhibit more similar online food preferences.

\section{TEMPORAL PATTERNS}

To identify differences in online food preferences over the course of a week, we compute for each nutrient its z-score to mitigate the effect of anomalous days [3]. Figure 1 shows the prevalence of sample nutrients ordered by group (meat, carbohydrates, fish, vegetables and alcohol). One can see that different groups of nutrients indeed reveal similar temporal trends regarding their popularity. Meat (e.g., pork and steaks) is mainly popular during the weekend with a peak on Sunday. This confirms offline observations (gained via questionnaires) which reveal that Austrians consume meat products more frequently on Sundays compared to other days in the week [2]. Carbohydrate-rich, cheap and healthy food such as pasta, vegetables and potatoes is more frequently

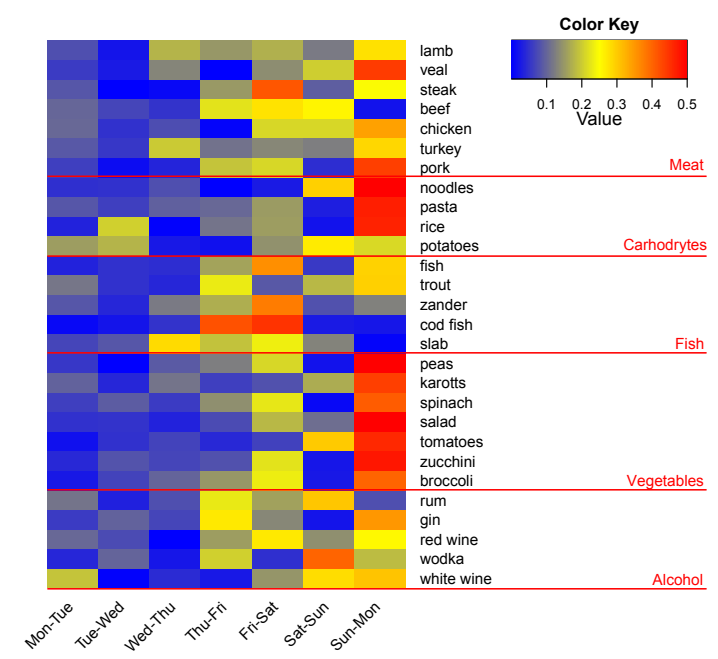

Figure 2: How do preferences change over the course of a week? The plot shows the average change rate from one day to the next one.

requested at the beginning of the week and less frequently during weekends. We also observe that fish is mainly requested on Thursday and Friday and alcohol is more popular at weekends than during the week.

Our preliminary observations suggest that online food preferences of users shift from weekdays to weekends. To further explore this hypothesis, we compute the average popularity change rate of two consecutive days $d_{i}$ and $d_{i+1}$ as follows:

$$
R\left(d_{i}, d_{i+1}\right)=\frac{\left|F\left(d_{i}\right)-F\left(d_{i+1}\right)\right|}{\sum_{j=1}^{N}\left|F\left(d_{j}\right)-F\left(d_{j+1}\right)\right|}
$$

$N$ is 7 since we sum over all consecutive weekday pairs and $F(d)$ refers to the access frequency at day $d$. Figure 2 shows that indeed most changes happen before and after the weekend, suggesting that online food preferences during the week are starkly different from weekend preferences. Changes in online food preferences over the course of a week slowly accumulate, with noticeable changes starting around Thu/Fri. The end of the weekend period is clearly demarcated, evident in a high change rate across most ingredients on Sun-Mon. This means that online food preferences tend to change slowly towards weekend preferences during the week, but they change abruptly back to weekday preferences over Sun-Mon.

Acknowledgements. We thank ichkoche.at for sharing their data with us.

\section{REFERENCES}

[1] E. N. Anderson. Everyone eats. Understanding food and culture. New York University Press, New York, London, 2005.

[2] I. Kiefer, C. Haberzettl, and C. Rieder.

Ernährungsverhalten und einstellung zum essen der österreicherinnen. Journal für Ernährungsmedizin, $2(5): 2-7,2000$.

[3] R. West, R. W. White, and E. Horvitz. From cookies to cooks: Insights on dietary patterns via analysis of web usage logs. In Word Wide Web conference ( $W W W)$, 2013. 